\title{
Anomalous Behavior of Adenovirus L4-33K Mutant Protein
}

\author{
Mohammad Feraz Ahsan* \\ Department of Medical Biochemistry and Microbiology, Uppsala University, \\ S-75123 Uppsala, Sweden \\ *Corresponding Author: Mohammad Feraz Ahsan, Department of Medical \\ Biochemistry and Microbiology, Uppsala University, S-751 23 Uppsala, Sweden. \\ E-mail: feraz.niv@gmail.com
}

\author{
Received: March 14, 2020 \\ Published: April 16, 2020 \\ (C) All rights are reserved by Mohammad \\ Feraz Ahsan.
}

\begin{abstract}
The late genes of adenovirus are transcribed from the major late transcription unit (MLTU), generating mRNA through L1 to L5. L4-33K protein is RNA splicing factor responsible for early to late switch in L1 alternative splicing. The L1-52,55K mRNA is produced during both early and late after infection, whereas L1-IIIa mRNA at late phase. L4-33K when phosphorylated by DNA-dependent protein kinase (DNA-PK) has an inhibitory effect on the temporal switch in L1 alternative RNA splicing, whereas phosphorylation with protein kinase A (PKA) has an enhancer effect on L1-IIIa splicing. The C-terminal region of L4-33K is conserved and responsible for splicing, nuclear localization and other functional activities. Mutational studies replacing serine with glycine in L4-33K, referred to as S176G, S189G, S196G did not hamper above functionality, but mutation at position 192 abolished the L4-33K splicing enhancer activity and nuclear localization. Here we show the posttranslational effect of these proteins against DNA-PK and PKA; all the mutants showed hypophosphorylation with DNA-PK and PKA, except S192G, which unusually showed hyperphosphorylation up to 2.63 times of the wild type L4-33K against DNA-PK, justifying earlier findings. This unusual result may be due to drastic misfolding of S192G exposing other phosphorylating residues. It would be interesting to carry out structural analysis of this protein during native and phosphorylated state.
\end{abstract}

Keywords: Adenovirus; L4-33K; Gene Regulation; RNA Splicing; Posttranslational Modification; DNA-PK; PKA

\section{Abbreviation}

CRE: cAMP-Responsive Element; CREB: cAMP-Responsive Element Binding Protein; DNA-PK: DNA-Dependent Protein Kinase; dsDNA: Linear Double Stranded DNA; DTT: Dithiothreitol; EBV: EpsteinBarr virus; MLP: Major Late Promoter; MLTU: Major Late Transcription Unit; PKA: Protein Kinase A

\section{Introduction}

Gene expression in Adenovirus is an extremely regulated and coordinated system expressing sequentially during infection. L4$33 \mathrm{~K}$ protein has been shown to functions as virus encoded alternative RNA splicing factor that regulates alternative splicing. The late proteins of adenovirus are translated from MLTU, extending from the major late promoter (MLP) till the termination signal. The pre-mRNA is 28,000 nucleotides expressed through MLTU, polyadenylated at specific sites giving rise to five families of mRNAs possessing co-terminal 3' ends (L1-L5) (Figure 1). Selecting polyA site, primary transcript undergoes splicing, where each mature mRNA receives a common tripartite leader segment which is spliced to several alternative 3 ' splice sites leading to more than 20 cytoplasmic mRNAs, subjected to a temporal regulation [1]. Same MLP promoter is utilized both during early and late phase of infection [2]. Early transcription through MLP decreases gradually after the L1 unit with fewer RNA polymerases extends beyond the L3 polyA sequence; whereas, at late phase the elongation continues downstream [3].

L1 is only unit of MLTU which produce mRNA both during early and late phase of infection. Temporally regulated splicing of L1 results in 52,5K mRNA from proximal and IIIa mRNA from distal ends. Interestingly, 52,55K mRNA is produced during early and late phase, whereas, IIIa mRNA only at late phase of infection. The L4 unit encodes four different mRNAs, of which L4-33K under study is one of them (Figure 1), a key regulators of adenoviral late gene expression, besides being engaged in packaging of the viral genome [4-6].

L4-33K undergoes flip in functionality based on phosphorylation by DNA-dependent protein kinase (DNA-PK) and cAMP dependent protein kinase (PKA). DNA-PK is a nuclear serine/threonine protein kinase from the family of phosphatidylinositol 3-kinaselike kinases (PIKKs) [7]. Usually phosphorylation by DNA-PK substrates is activated by linear double stranded DNA (dsDNA) [8]. DNA-PK is a heterotrimeric enzyme with a catalytic subunit (DNAPKcs) and two regulatory subunits Ku70 and Ku86 [9]. Besides the DNA repair, it can interact and phosphorylate the C-terminal domain of RNA Pol II, p53, Sp1, c-Myc, c-Jun and other transcription factors, thereby regulating the initiation of transcription $[10,11]$. 
Similarly, PKA has been found to regulate the gene expression by phosphorylation of many transcription factors like cAMP-responsive element (CRE) binding protein (CREB) [12]. It requires cAMP for activation, else it is inactive tetramer with two regulatory $(\mathrm{R})$ and two catalytic $(\mathrm{C})$ subunits. PKA activates upon binding of four cAMP molecule to the $\mathrm{R}$ dimer, releasing $\mathrm{C}$ subunits to phosphorylate the serine and threonine residues of substrate [13] The activated $\mathrm{C}$ subunits can translocate to the nucleus which can regulate pre-mRNA splicing by phosphorylating the splicing factors [14].

In case of Adenovirus, studies have shown that L4-33K gets highly phosphorylated by DNA-PK in a double stranded DNA independent manner and have inhibitory role of DNA-PK in temporal switch of L1 alternative splicing of RNA. Inversely when L4-33K is phosphorylated by PKA, it induced L1-IIIA splicing, thus both these kinases regulate RNA splicing by L4-33K with inverse effects [15]. In another study it has been found that L4-33K protein localize and reorganize as viral transcription site to the nucleus in late virus infected cells, but mutant S192G hampered the nuclear localization of protein [16].

The functional significance of L4-33K and the effect of mutation at position 192 has been extensively focused, but the posttranslational modification of this protein is unknown. Hence, we further extended our comparative study with all the sets of mutants (refer: methods) against both DNA-PK and PKA and discussed the intriguing effect of phosphorylation on S192G with DNA-PK.

\section{Materials and Methods}

Plasmids

L4-33K protein of Adenovirus and its mutants S176G, S189G, S192G, S196G and DS were used in the studies as described and used elsewhere (Figure 1) $[4,15,16]$. Briefly, amplicons were generated through RT-PCR using late cytoplasmic RNA from wild type Ad5-infected cells, mutants were developed using PCR based mutagenesis to construct the deletion and substitution mutants of L4$33 \mathrm{~K}$ replacing serine to glycine at aforesaid position, and 27 amino acid deleted from carboxy terminal for DS mutant. Wild type L4$33 \mathrm{~K}$ protein was mentioned as wtL4-33K. L4-33K and its variants were overexpressed in bacterial expression system through pET24a (Novagen), resulting in the production of aforementioned proteins containing a C-terminal His-tag [4].

\section{Protein expression and purification}

L4-33K and its mutants protein were expressed and purified as described in the earlier publication from our lab [4]. Briefly, $E$. coli BL21 (DE3) transformed cells with 0.1 mM IPTG were induced and after sufficient growth, cells were lysed in lysis buffer $(20 \mathrm{mM}$ Hepes-KOH pH 7.9, $300 \mathrm{mM} \mathrm{KCl,} \mathrm{0.05 \%} \mathrm{Triton} \mathrm{X-100)} \mathrm{in} \mathrm{the} \mathrm{pres-}$ ence of protease inhibitor (Complete Mini EDTA free, Roche Applied sciences) using sonicator (Bioruptor, Diagenode). The pro- teins of interest were recovered from lysates using standard nickel column chromatography (Poly-Prep column, Biorad). The eluted proteins were dialyzed against Buffer D (20 mM Hepes, pH 7.9, $20 \%$ glycerol, $0.1 \mathrm{M} \mathrm{KCl}, 0.2 \mathrm{mM}$ EDTA pH 8, $0.5 \mathrm{mM}$ dithiothreitol (DTT) and stored at $-20^{\circ} \mathrm{C}$. Protein concentration was determined using Bradford method using bovine serum albumin as a standard [17].

\section{Kinase assay}

The kinase assay was performed with wtL4-33K and mutants as previously mentioned for DNA-PK and PKA with some modifications [17]. Briefly $0.5-3 \mu \mathrm{g}$ of substrate protein wtL4-33K and all its mutants (each set for DNA-PK and PKA) were used up in the reaction buffer resulting in the final concentration of $50 \mathrm{mM}$ Hepes$\mathrm{KOH} \mathrm{pH} \mathrm{7.9,} \mathrm{0.1M} \mathrm{KCl,} 10 \mathrm{mM} \mathrm{MgCl}_{2}$, 1 mM DTT, 0.1 mM EDTA pH 8, $0.2 \mathrm{mM}$ ATP, $1.2 \mu \mathrm{g}$ BSA, $2 \mu \mathrm{Ci}$ of $\gamma^{32} \mathrm{P}$-ATP and $0.15 \mu \mathrm{g}$ linear doublestranded DNA (HpaII cleaved purified pUC19 (Fermentas)) plasmid DNA). All components were mixed and incubated 3 minutes at $30^{\circ} \mathrm{C}$. The enzyme DNA-PK and PKA $(10 \mathrm{u})$ respectively was added in the reaction and incubated at $30^{\circ} \mathrm{C}$ for 15 minutes. Reactions were terminated by adding Laemmli buffer and heated at $95^{\circ} \mathrm{C}$ for $10 \mathrm{~min}-$ utes, proteins were separated on 12\% SDS-PAGE gel and stained using CBB. The incorporation $\gamma^{32}$ P-ATP was analysed by PhosphorImager (BioRad). The results were normalized against wtL4-33K and evaluated densitometrically using ImageJ.

\section{Results}

Phosphorylation analysis of wtL4-33K and its mutants against DNA-PK and PKA

L4-33K protein was characterized as an interesting multifunctional phosphoprotein involved in several aspects of viral gene expression, still lacks the information on posttranslational modifications $[4,5,18-20]$. To further understand the complexities we used the previously studied sets of L4-33K mutants (Figure 1) [4], viz. wtL433K, S176G, S189G, S192G, S196G and DS.

Studies indicated that L4-33K protein to be a substrate for DNA-PK and PKA phosphorylation. To get further insight into the protein, several kinase assays were performed with the purified L4-33K proteins which suggested it to be efficiently phosphorylated by both DNA-PK and PKA (Figure 2a, $2 \mathrm{~b}$ and $3 \mathrm{a}$ and $3 \mathrm{~b}$ ). Phosphorylation by DNA-PK showed reduced phosphorylation of all the mutants when compared to wtL4-33K at varying level, except for S192G which unusually showed increased phosphorylation up to 2.63 times (Figure 2a and 2b). Whereas, phosphorylation by PKA was shown to decrease the phosphorylation to varying degree for all the mutants when compared to wtL4-33K (Figure 3a and 3b). This finding suggests that S192G is hyperphosphorylated by DNA-PK in an unusual manner. The result shown here is the representative of many independent experiments which showed very consistent results for many fold hyperphosphorylation exclusively for S192G when treated with DNA-PK, whereas all other mutant 


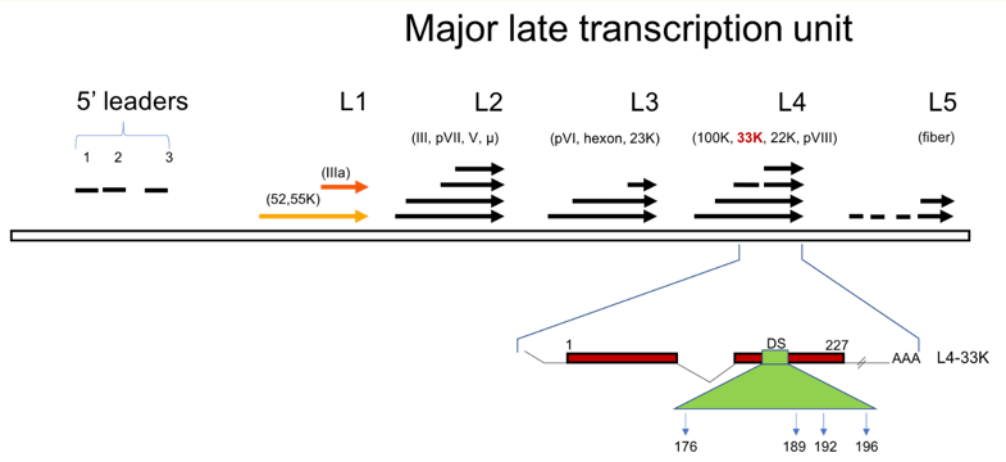

Figure 1: Schematic representation of spliced structure of major late transcription unit. The tri-partite 5' leader is included in all mRNAs expressed from the MLTU. The 52,55K and IIIa mRNA due to active interaction with L4-33K is distinctly shown in coloured. The functionally important region as mutants under study are magnified.

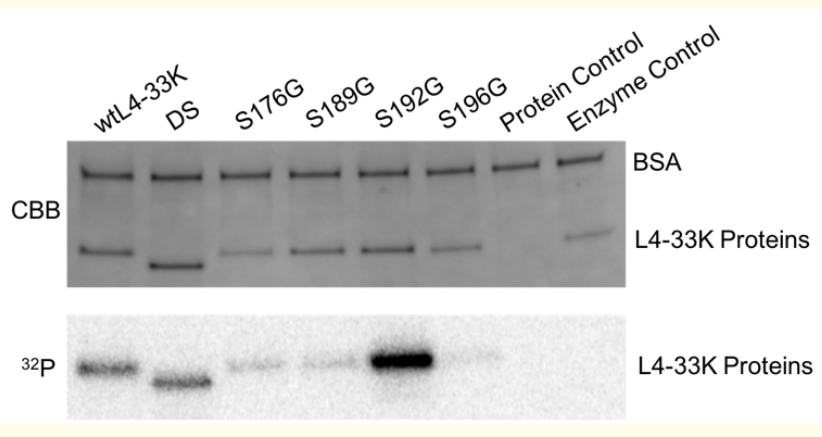

Figure 2a: The wild type L4-33K and mutants resulting after kinase assay with DNA-PK. Proteins were resolved by gel electrophoresis followed by CBB staining and incorporation of radiolabelled phosphate was visualized by autoradiography.

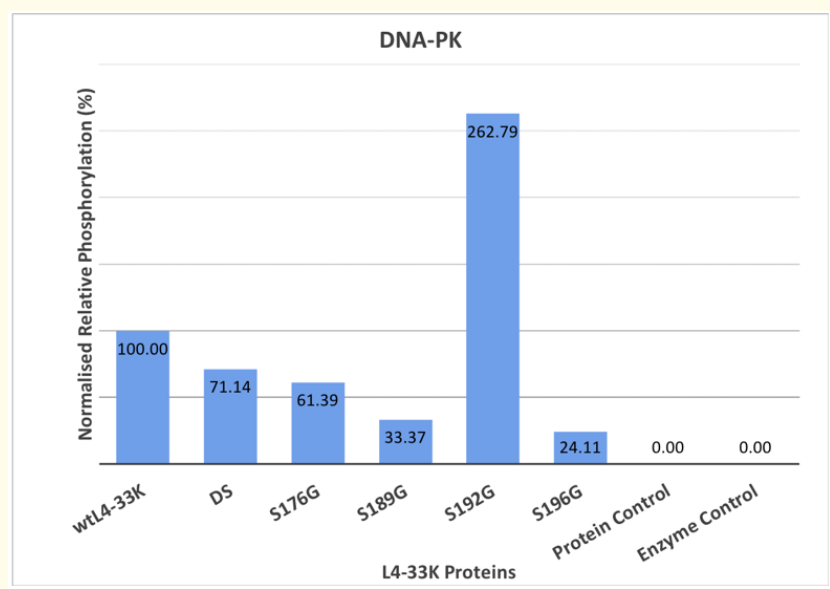

Figure 2b: The value in the graph represents the incorporated phosphorylation for each of the mutants and wild type L4-33K derived from the result as shown in figure 2a against DNA-PK. The level of phosphorylation were quantified, normalised against wtL4-33K and plotted as percent relative phosphorylation.

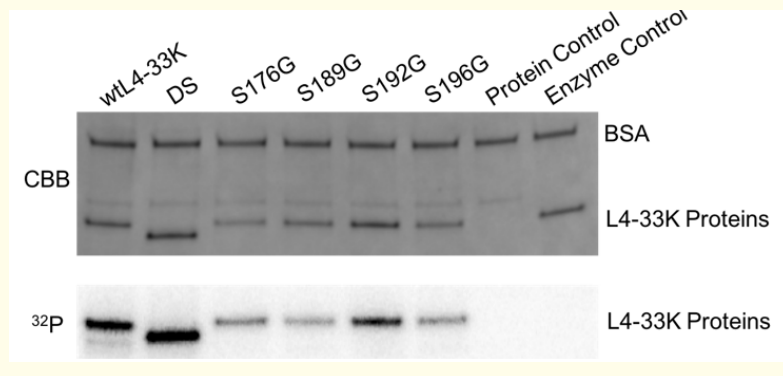

Figure 3a: The wild type L4-33K and mutants resulting after kinase assay with PKA. Proteins were resolved by gel electrophoresis followed by CBB staining, and incorporation of radiolabelled phosphate was visualized by autoradiography.

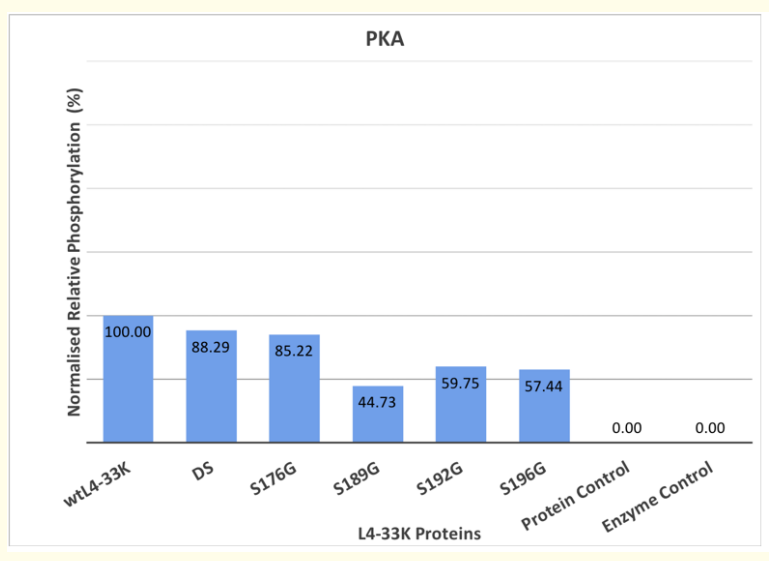

Figure 3b: The value in the graph represents the incorporated phosphorylation for each of the mutants and wild type L4-33K derived from the result as shown in figure $3 \mathrm{a}$ against PKA. The level of phosphorylation were quantified, normalised against wtL4-33K and plotted as percent relative phosphorylation. 
showed decrease in the activity when treated with either DNAPKA or PKA (representative result is presented here, hence, statistical analysis has not been done). Due to variation in the input protein for the wtL4-33K and its mutants, the phosphorylated value for each mutant was normalized against wtL4-33K, which was considered as 100 percent relative phosphorylation.

\section{Discussion}

The earlier studies from our lab have confirmed the novel interplay of Adenovirus L4-33K protein and phosphorylation effect by DNA-PK and PKA in regulation of late gene alternative splicing. The first report on phosphoprotein of L4-33K protein connected to kinase activity was dissected by Perssson., et al. in 2012 for demonstrating the two cellular protein kinases with opposite effects on functionality of L4-33K protein [15]. L4-33K protein was shown to be highly phosphorylated by DNA-PK in vitro, it notably blocks the early to late switch in adenoviral L1-52,55K/IIIa mRNA splicing, pointing out DNA-PK as a regulator of adenovirus MLTU alternative splicing. L4-33K is also phosphorylated by PKA which has enhancing effect on L4-33K- activated L1-IIIa splicing. Collectively, both DNA-PK and PKA phosphorylate L4-33K, having distinct and opposite effects on early to late shift in L1 alternative splicing. The study also supported that phosphorylation site of DNA-PK resides at the unique C-terminal domain of L4-33K [15]. It was also shown by others that PKA have marked effect on L1 mRNA accumulation, because L4-33K activated splicing of L1-IIIa pre-mRNA and also transcriptionally activate MLP [17]. Similar studies with EpsteinBarr virus (EBV) protein EBNA-LP were linked to have regulated with DNA-PK and PKA [21].

L4-33K is the only viral protein sufficient to mediate the temporal activation of major late pre-mRNA splicing during late times of infection, indicating it to be central viral component of multiprotein complex enhancing splicing [15]. Deleting 157 amino-terminal of L4-33K did not much reduce the splicing enhancer activity. The carboxy terminal of the protein is most conserved and functionally crucial as splicing enhancing domain. Mutating tiny RS repeat of serine residue at position 176, 189, 196 (Figure 1) did not show adverse effect on splicing, whereas, mutation at position 192 abolished the L4-33K splicing enhancer activity, which is the crucial observation of this study [15].

The study was further extended in our lab on functionality of L4-33K, that serine residue within RS repeat of C-terminus are important for nuclear localization and redistribution of L4-33K during late phase of infection, and serine at position 192 is pivotal for this functionality [16]. Mutant 192 failed to relocalize at viral replication centre in Ad5 infected cells, indicating functional role of RS repeat in assembly of virus replication machinery. The importance of serine at position 192 was further delineated through phosphomimetic mutation by replacing serine with aspartic acid, it still did not relocalize to the replication centres during infection and also rendered the protein inactive as L1 splicing factor, which further confirms the previous observations [16]. A similar study with lamin B receptor of human having short RS repeat and its phosphorylation leads to conformational change, which is important for lamin $B$ receptor binding to H3-histone [22].

The above findings emphasized on the functional significance of serine at position 192. The current study was continuum to our quest to understand posttranslational modification effect to unleash the role of serine 192 mutant in comparison to other mutants when treated with DNA-PK and PKA (Figure 1). Point mutation studies are commonly used methodology to map any vital amino acid for functional significance. We used the same strategy to study phosphorylation of individual mutant and observed a decrease in phosphorylation (except S192G) in comparison to wtL4-33K at varying level for both DNA-PK and PKA. Due to lack of functional significance as per earlier studies with these mutants, magnitude of hypophosphorylation is not being discussed. In theory, the mutant S192G treated with DNA-PK/PKA was expected to hypophosphorylate, as depicted in the light of its functional activity. Surprisingly, S192G hyperphosphorylated to a great extent with DNA-PK against the paradigm of expected observation. Possibly, mutation at position 192 drastically misfolded the protein, exposing other phosphorylating amino acid residues through DNA-PK, resulting in hyperphosphoryltion, whereas PKA apparently follows different phosphorylation pattern [23]. Alternatively, phosphorylated serine at position 192 in wtL4-33K may be crucial for proper folding of protein and maintaining the splicing activity of L4-33K, but loss of phosphorylation in S192G deformed the protein structure/ active site resulting in the loss of functional activity. A recent study with hepatitis c virus showed that phosphorylation of specific residue can lead to sequential phosphorylation leading to hyperphosphorylation, which might be another indirect possibility in this case [24]. Numerous hypothesis and physiochemical possibilities remains open to solve the complexities of such mechanism, alternatively this modality could even lead to new findings about DNA-PK and PKA [25].

This study requires the structural assessment of this protein, it would be fascinating to compare the structural transformations in wtL4-33K and S192G during native and phosphorylated state, which could further clarify our understanding. This astounding behaviour of S192G mutants is unravelling more questions than answers in understanding the protein behaviour. The lesson learnt from this investigation could be extended in understanding posttranslational effect of point mutation and physiological consequences, protein-protein interaction, exploring biomarker to target protein, and also consider these possibilities during study design for proteomic analysis. 
This is the first report on hyperphosphorylation of a mutant protein exceeding the wild type in the above context. The study highlights the tangential perspective of a mutant viral protein, which could be of immense importance to open the avenue for more serious investigations.

\section{Conclusion}

This current study was to understand the posttranslational effect on the adenoviral L4-33K mutants phosphorylated against DNA-PK and PKA. The noteworthy finding was S192G mutant hyperphosphorylated against DNA-PK, possibly as a consequence of drastic conformational change; whereas all other mutants showed hypophosphorylation for both DNA-PK and PKA. This study could not present details about structural information highlights for hyperphosphorylation of S192G. Still it shed some light on posttranslational modification of mutant which has affected the functionality. The further study on detailed structural analysis of S192G and wtL4-33K both as a native and phosphorylated state against DNAPK and PKA would be interesting to elucidate.

\section{Acknowledgements}

I am grateful to Prof. Göran Akusjärvi for giving me the opportunity to work in his lab and provide expert guidance during study. The research presented here was made possible by generous grants from the Swedish Cancer Society and the Swedish Research Council to G. Akusjärvi.

The current address of author is: IUCBR\&SSH, MG University, Kottayam, Kerala, India.

\section{Conflict of Interest}

There is no conflict of interest.

\section{Bibliography}

1. Biasiotto R and Akusjärvi G. "Regulation of human adenovirus alternative RNA splicing by the adenoviral L4-33K and L422K proteins". International Journal of Molecular Sciences 16.2 (2015): 2893-2912.

2. Shaw AR and Ziff EB. "Transcripts from the adenovirus-2 major late promoter yield a single early family of 3' coterminal mRNAs and five late families". Cell 22.3 (1980): 905-916.

3. Iwamoto S., et al. "Transcription unit mapping in adenovirus: regions of termination". Journal of Virology 59.1 (1986): 112119.

4. Törmänen $\mathrm{H}_{\text {., }}$ et al. "L4-33K, an adenovirus-encoded alternative RNA splicing factor". Journal of Biological Chemistry 281.48 (2006): 36510-36517.

5. Farley DC., et al. "Activation of the Early-Late Switch in Adenovirus Type 5 Major Late Transcription Unit Expression by L4 Gene Products". Journal of Virology 78.4 (2004): 1782-1791.
6. Ewing SG., et al. "Ternary Complex Formation on the Adenovirus Packaging Sequence by the IVa2 and L4 22-Kilodalton Proteins". Journal of Virology 81.22 (2007):12450-12457.

7. Lempiäinen $\mathrm{H}$ and Halazonetis TD. "Emerging common themes in regulation of PIKKs and PI3Ks". The EMBO Journal 28.20 (2009): 3067-3073.

8. Carter T., et al. "A DNA-activated protein kinase from HeLa cell nuclei". Molecular and Cellular Biology 10.12 (1990): 64606471.

9. Yaneva M., et al. "Interaction of DNA-dependent protein kinase with DNA and with $\mathrm{Ku}$ : biochemical and atomic-force microscopy studies". The EMBO Journal 16.16 (1997): 5098-5112.

10. Maldonado E., et al. "A human RNA polymerase II complex associated with SRB and DNA-repair proteins". Nature 381.6577 (1996): 86-89.

11. Lees-Miller SP. "The DNA-dependent protein kinase, DNA-PK: 10 years and no ends in sight". Biochemistry and Cell Biology 74.4 (1996): 503-512.

12. Montminy M. "Transcriptional regulation by cyclic AMP". Annual Review of Biochemistry 66.1 (1997): 807-822.

13. Rannels SR and Corbin JD. "Two different intrachain cAMP binding sites of cAMP-dependent protein kinases". Journal of Biological Chemistry 255.15 (1980): 7085-7088.

14. Nigg EA., et al. "Rapid and reversible translocation of the catalytic subunit of cAMP-dependent protein kinase type II from the Golgi complex to the nucleus". The EMBO Journal 4.11 (1985): 2801-2806.

15. Törmänen Persson H., et al. "Two cellular protein kinases, DNA-PK and PKA, phosphorylate the adenoviral L4-33K protein and have opposite effects on L1 alternative RNA splicing". PLoS One 7.2 (2012): e31871.

16. Östberg S., et al. "Serine 192 in the tiny RS repeat of the adenoviral L4-33K splicing enhancer protein is essential for function and reorganization of the protein to the periphery of viral replication centers". Virology 433.2 (2012): 273-281.

17. Howland JL. "Short protocols in molecular biology, third edition. Edited by F Ausubel, R Brent, R E Kingston, D D Moore, J G Seidman, J A Smith and K Struhl". Biochemical Education 24.1 (1996): 68-68.

18. Morris SJ and Leppard KN. "Adenovirus Serotype 5 L4-22K and L4-33K Proteins Have Distinct Functions in Regulating Late Gene Expression". Journal of Virology 83.7 (2009): 3049-3058. 
19. Finnen RL., et al. "Truncation of the human adenovirus type 5 L4 33-kDa protein: evidence for an essential role of the carboxy-terminus in the viral infectious cycle". Virology 289.2 (2001): 388-399.

20. Fessler SP and Young CSH. "The role of the L4 33K gene in adenovirus infection". Virology 263.2 (1999): 507-516.

21. Han I., et al. "EBNA-LP Associates with Cellular Proteins Including DNA-PK and HA95". Journal of Virology 75.5 (2001) 2475-2481.

22. Sellis D., et al. "Phosphorylation of the arginine/serine repeats of lamin B receptor by SRPK1-insights from molecular dynamics simulations". Biochimica et Biophysica Acta 1820.1 (2012): 44-55.

23. Summers KC., et al. "Phosphorylation: the molecular switch of double-strand break repair". International Journal of Proteomics (2011): 373816.

24. Pan T-C., et al. "Differential Proteomics Reveals Discrete Functions of Proteins Interacting with Hypo- versus Hyper-phosphorylated NS5A of the Hepatitis C Virus". Journal of Proteome Research 18.7 (2019): 2813-2825.

25. Nishi H., et al. "Physicochemical mechanisms of protein regulation by phosphorylation". Frontiers in Genetics 5 (2014): 270

\section{Assets from publication with us}

- Prompt Acknowledgement after receiving the article

- Thorough Double blinded peer review

- Rapid Publication

- Issue of Publication Certificate

- High visibility of your Published work

Website: https://www.actascientific.com/

Submit Article: https://www.actascientific.com/submission.php Email us: editor@actascientific.com

Contact us: +919182824667 